\title{
Orientational dynamics in methanol: influence of temperature and hydrogen bonding
}

\author{
Jordi Ortiz de Urbina and Gemma Sesé* \\ Departament de Física, Universitat Politècnica de Catalunya, Campus Nord-Mòdul B4, \\ c/ Jordi Girona 1-3, 08034 Barcelona, Spain
}

\begin{abstract}
Rotational diffusion has been investigated in methanol by means of molecular dynamics simulations at several temperatures, approaching the supercooled state. Angular velocity autocorrelation functions in the molecule-fixed coordinate frame have been evaluated, and the principal components of the rotational diffusion tensor have been obtained. Rotational dynamics of molecules belonging to different hydrogen-bonded states has also been analyzed. It has been found that out-of-plane rotation is favoured at room temperature. Upon cooling, out-of-plane rotational diffusion coefficients decrease faster than inplane ones, which become largest in the supercooled state. Nevertheless, differences on the microscopic leading mechanisms of rotational diffusion at low temperatures have been identified. For molecules that establish only one hydrogen bond, out-of-plane dynamics is still the most relevant in the supercooled state. Rotational relaxation has also been studied and the available theoretical models have been tested.
\end{abstract}

Keywords: Molecular Dynamics, Methanol, Rotational diffusion, Reorientation

\section{Introduction}

A detailed understanding of the dynamics of alcohols at a molecular level is of paramount relevance because of their role as solvents in many chemical processes. It is generally accepted that their hydrogen bonding network strongly influences thermodynamic, structural and dynamic properties $[1$,

*gemma.sese@upc.edu 
$2,3]$. Computer simulation has proved to be an invaluable tool to analyze the microscopic processes leading to macroscopic properties. It has been also used to investigate methanol dynamics $[4,5]$ as well as the role that hydrogen bonds play on single particle properties related to the dynamics of translation $[6,7]$. We intend to extend that analysis to rotational dynamics.

The Debye rotational diffusion model [8] describes reasonably well the dynamics of non-associated liquids by considering that their molecules perform rotational Brownian motions [9]. It has been previously shown that methanol is a non-Debye fluid at room temperature [10], but its deviations from the Debye model for reorientation characteristic times are smaller than those obtained for water [11]. The extended jump model has proven to be successful in the analysis of molecular reorientation in hydrogen bonded liquids. The approach works particularly well for water, where reorientation occurs through large-amplitude angular jumps which lead to a change of the specific hydrogen bond (HB) partner $[12,13]$. According to the model, reorientation characteristic times include the contribution of the time of a jump as well as that of the time spent between jumps. When the extended jump model was applied to methanol at room temperature, it was found that reorientation of intact HB is the dominant microscopic mechanism over jumps leading to hydrogen exchange. This behaviour is qualitatively different from that observed in water [14]. Our study is intended to provide some insight onto the diffusion mechanisms concerning rotational dynamics of molecules while the hydrogen bonds that they establish are unchanged, a goal which is beyond the scope of the model. It seems reasonable that such mechanisms depend on the bonding state of molecules, that is, on the number of HB that a molecule establishes. Then, different bonding states will be analyzed.

The main purpose of this work is to contribute to the study of rotational diffusion in methanol by using molecular dynamics experiments. In order to analyze anisotropy of rotational diffusion, angular velocity time autocorrelation functions about the molecular principal axes of inertia have been computed. They have been used to obtain the rotational diffusion tensor in the molecular reference frame. Rotational diffusion tensors of methanol molecules at specific hydrogen bonded states have also been evaluated. Even though these coefficients cannot be directly compared to experimental data, they can shed some light onto the microscopic dynamics of the molecular model.

Reorientational correlation functions of the dipole moment in the laboratory reference frame have also been obtained at several temperatures. Some 
theoretical approaches on rotational relaxation have been tested. They relate characteristic times evaluated from angular velocity autocorrelation functions and those associated with dipole moment reorientation [15]. More specifically, predictions of the extended diffusion models [16] and the Langevin model [17] have been analyzed.

\section{Methods}

Molecular dynamics simulations of methanol have been performed. A three-site molecular model has been used. Sites $\left(\mathrm{CH}_{3}, \mathrm{O}\right.$ and $\left.\mathrm{H}\right)$ are connected through rigid bonds whose length is constrained using the SHAKE algorithm [18]. Molecular sites belonging to different molecules interact by means of electrostatic and Lennard-Jones forces according to the OPLS potential [19]. The model provides realistic results for static and dynamic properties of methanol [21]. The Ewald summation [20] has been used in the evaluation of electrostatic interactions. The simulated samples are made up of 1000 molecules located in a cubic box with periodic boundary conditions.

Reorientational dynamics has been analyzed by means of the $C_{l}(t)$ correlation function, defined as

$$
C_{l}(t)=<P_{l}(\mu(\mathbf{t}) \cdot \mu(\mathbf{0}))>,
$$

where $P_{l}$ refers to the $l$ th Legendre polynomial and $\mu(\mathbf{t})$ is the unit vector in the direction of the molecular dipole moment after a time interval $t$ [20]. Previous results have shown that the long-time part of the $C_{l}(t)$ function can be fitted to a stretched exponential, being $\tau_{l}$ the associated correlation time or reorientational time [2] .

For non-isotropic molecular systems, the directional dependence of rotational motions can be analyzed by using autocorrelation functions of angular velocities around the molecular principal axes of inertia

$$
C_{\omega_{k}}(t)=\frac{<\omega_{k}(t) \cdot \omega_{k}(0)>}{<\omega_{k}(0) \cdot \omega_{k}(0)>}
$$

where $k$ refers to any of the three principal inertial axes. The principal inertial axes for a three-site methanol molecule are plotted in figure 1. The $\mathrm{x}$ axis is perpendicular to the molecular plane. Then, $C_{\omega_{x}}(t)$ functions give information about in-plane dynamics, whereas $C_{\omega_{y}}(t)$ and $C_{\omega_{z}}(t)$ refer to the out-of-plane motions. The associated correlation times $\left(\tau_{k}\right)$ can be evaluated by numerical integration of these functions. 
$C_{\omega_{k}}(t)$ functions have been evaluated for different hydrogen bonded states. We have employed a widely used criterion for determining HB partners that relies on geometrical analyses $[21,22]$. It states that a $\mathrm{HB}$ is established between two molecules if $d_{O H} \leq 2.6 \AA, d_{O O} \leq 3.5 \AA$, and if the angle (HO $\cdots \mathrm{O}) \leq 30^{\circ}$. HB lifetimes increase dramatically upon cooling. We have found that continuous HB lifetimes are about 1.4 ps at room temperature, whereas they increase up to $56 \mathrm{ps}$ at $178 \mathrm{~K}$. $C_{\omega_{k}}(t)$ functions have been evaluated for time intervals up to $3 \mathrm{ps}$, as angular velocities become decorrelated on the subpicosecond timescale, even at low temperatures. Then, it is extremely unlikely that a molecule changes its $\mathrm{HB}$ state within those time intervals. Production runs of $200 \mathrm{ps}$ at high temperatures and of $300 \mathrm{ps}$ at the lowest temperature have been used to obtain these functions. Longer runs of $1 \mathrm{~ns}$ and 6 ns respectively have been required to evaluate $C_{l}(t)$, because of their large characteristic times.

As not all the directions are equivalent, the rotational diffusion coefficient becomes a second rank tensor, represented by a square $3 \times 3$ matrix. The diagonal terms of the rotational diffusion tensor can be obtained as [23, 24].

$$
D_{k}^{\omega}=\int_{0}^{\infty}<\omega_{k}(0) \cdot \omega_{k}(0)>C_{\omega_{k}}(t) d t
$$

or, alternatively, from the slope of the mean squared orientational displacement [25]

$$
D_{k}^{\theta}=\frac{1}{2 t}<\Delta \theta_{k}(t) \cdot \Delta \theta_{k}(0)>
$$

These functions can be related to the angular momentum time correlation function $[16,26]$, which can be defined as

$$
C_{J}(t)=\frac{<\mathbf{J}(\mathbf{t}) \cdot \mathbf{J}(\mathbf{0})>}{<\mathbf{J}(\mathbf{0}) \cdot \mathbf{J}(\mathbf{0})>}
$$

Its associated relaxation time $\tau_{J}$ is the characteristic time between collisions, which encompasses randomization of angular momentum. It can be evaluated from $\tau_{k}$ through

$$
\tau_{J}=\frac{\omega_{x}(0)^{2} \cdot \lambda_{x}^{2} \cdot \tau_{x}+\omega_{y}(0)^{2} \cdot \lambda_{y}^{2} \cdot \tau_{y}+\omega_{z}(0)^{2} \cdot \lambda_{z}^{2} \cdot \tau_{z}}{\omega_{x}(0)^{2} \cdot \lambda_{x}^{2}+\omega_{y}(0)^{2} \cdot \lambda_{y}^{2}+\omega_{z}(0)^{2} \cdot \lambda_{z}^{2}}
$$

where $\lambda_{x}=17.6 \mathrm{uma} \cdot \AA^{2}, \lambda_{y}=16.9 \mathrm{uma} \cdot \AA^{2}$ and $\lambda_{z}=0.74 \mathrm{uma} \cdot \AA^{2}$ are the diagonal terms of the inertial tensor for the considered methanol model. Most 
theories on rotational relaxation are concerned with the relationship between characteristic times $\tau_{J}$ and $\tau_{l}$.

\section{Results}

\subsection{Rotational spectra}

Measurements performed by far infrared spectroscopy have identified three bands at about 130, 280 and $570 \mathrm{~cm}^{-1}$ in liquid methanol [27]. Based on comparisons with experiments on deuterated methanol, the lowest and the highest bands were assigned to librations about axes perpendicular and parallel to the $\mathrm{C}-\mathrm{O}$ bond respectively. The intermediate band was assigned to translational motions via the dipole induced dipole mechanism, which is not allowed by our model. Simulation allows to check whether these modes depend on the specific hydrogen bonded state.

Autocorrelation functions of angular velocities about the principal inertial axes have been evaluated for different subsets of molecules, corresponding to different hydrogen bonded states. As shown in figure 1, the $\mathrm{x}$ axis is perpendicular to the molecular plane. Fourier transforms of $C_{\omega_{x}}(t)$ at room temperature are displayed in figure 2. It is shown that the zero frequency value decreases as the number of $\mathrm{HB}$ increases, which is associated with decreasing rotational diffusion coefficients. For 2-HB and 3-HB molecules, a wide band appears around $170 \mathrm{~cm}^{-1}$, whereas a peak centered at 155 $\mathrm{cm}^{-1}$ is apparent for 1-HB molecules. For non-associated molecules, a band is observed at about $120 \mathrm{~cm}^{-1}$. Then, our results permit to associate the low frequency band observed in experiments with in-plane rotations of both bonded and non-bonded molecules.

Fourier transforms of $C_{\omega_{y}}(t)$ at room temperature are shown in figure 3. They can be related to the out-of-plane rotation of the methyl site (see figure 1). It is apparent that out-of-plane rotational dynamics takes place at shorter frequencies than in-plane rotations. A low frequency maximum had been observed in several non-associated dense liquids [6], which was typically assigned to rattling translational motions of molecules within the cage formed by their neighbours. A broad band which is apparent around 40 $\mathrm{cm}^{-1}$ in figure 3 for the non-HB molecules can be associated to the rattling rotational motion of methyl sites. As shown in figure 3, the peak shifts towards slightly increasing frequencies as the number of HB increases, and it is centered about $70 \mathrm{~cm}^{-1}$ for the 2-HB subset. Fourier transforms of $C_{\omega_{y}}(t)$ and $C_{\omega_{x}}(t)$ are displayed on the inset of figure 3. They have been normalized 
so that their values at zero frequency have been set to one, in order that the relative relevance of peaks could be estimated. It is apparent that the peak observed on the $y$ direction is less relevant than that corresponding to in-plane rotations ( $x$ direction). This would explain why the former does not appear in experiments as a separate peak, and it probably contributes to the band centered at $130 \mathrm{~cm}^{-1}$.

Fourier transforms of $C_{\omega_{z}}(t)$ at room temperature are shown in figure 4 . They give information about out-of-plane rotation of the hydrogen site (see figure 1), which takes place at increasing frequencies as the number of $\mathrm{HB}$ increases. The 1-HB subset displays a peak at about $580 \mathrm{~cm}^{-1}$ whereas a marked and wide peak at about $600 \mathrm{~cm}^{-1}$ characterizes the 2-HB subset. Both of them are very close to the experimental band observed at about 570 $\mathrm{cm}^{-1}$.

Upon cooling, the proportion of molecules with 2-HB increases from $75 \%$ at room temperature to $96 \%$ at $158 \mathrm{~K}$, whereas the proportion of molecules in 0 - $\mathrm{HB}$ decreases from $1 \%$ to zero, and those of 1 - HB and 3-HB decrease from $16 \%$ and $7 \%$ at $298 \mathrm{~K}$, respectively, to $2 \%$ at $158 \mathrm{~K}$ [5]. For 2-HB molecules, the largest proportion subset at all temperatures, Fourier transforms of $C_{\omega_{x}}(t)$ and $C_{\omega_{y}}(t)$ at different temperatures are shown in figure 5. It is apparent that main peaks are shifted towards slightly larger frequencies. Moreover, shoulders observed at room temperature develop into secondary peaks at the lower analyzed temperatures, specially for rotation about the $\mathrm{x}$ axis. Similar qualitative behaviour has been encountered for all HB subsets.

\subsection{Rotational diffusion tensor}

Diagonal elements of the rotational tensor at room temperature for molecules belonging to specific HB subsets $\left(D_{k}^{R}\right)$ are gathered in table 1 . They have been obtained by means of eq. (3). It should be noted that values corresponding to 2-HB methanol molecules are very similar to the ones of the overall system because of the large proportion of molecules belonging to this HB state. It is apparent that all $D_{i}^{R}$ decrease as the HB number increases, which is consistent with an increasingly constrained dynamics. In addition, diffusion about the $z$ axis is largest only for non-associated molecules, mainly because of the smaller mass of hydrogen sites. It is remarkable that rotational diffusion about the $y$ axis (out-of-plane) is largest for every HB subset. Rotation about $y$ axis reflects basically the dynamics of $\mathrm{CH}_{3}$ sites and it does not involve the breaking of any HB. Nevertheless, $D_{y}^{R}$ is lowest for 3HB molecules. Then, branching significantly hinders rotational dynamics of 
$\mathrm{CH}_{3}$ sites probably because the former diminishes the available free volume. On the contrary, it scarcely influences rotation about $x$ and $z$ axes, because of the highly constrained dynamics of hydrogen sites in 2HB-states, even at room temperature.

Diagonal elements of the rotational tensor at different temperatures are gathered in table 2. They have been evaluated by using eq.(3). Equation(4) has also been employed to calculate such coefficients, and the obtained results lie within the stated margin of error. It is apparent that all diagonal elements of the rotational tensor decrease upon cooling, but out-of-plane rotational diffusion coefficients (about $y$ and $z$ axis) decrease faster than inplane ones (about $x$ axis). Indeed, whereas diffusion about the $y$ axis is the predominant mechanism for rotation at room temperature, in-plane rotation is the most relevant one at very low temperatures. It was previously found that out-of-plane dynamics is favoured at room temperature for water, and that their associated rotational diffusion coefficients decrease at higher rates than their in-plane counterparts. As a result, in-plane rotation prevails at low temperatures, being the rationale behind this behaviour that rotation with the highest barrier stops first whereas rotation with the lowest energy barrier persists to the lowest temperatures [24]. For methanol, even though rotation about the $y$ axis does not require any HB breaking, large inertial effects on the $\mathrm{CH}_{3}$ dynamics, which are more difficult to overcome as temperature decreases, probably account for this behaviour. We also found that $D_{y}^{R}$ decreases by a factor of 50 for the whole system, where 2-HB molecules are the majority, but it decreases only by a factor of 10 for 1-HB molecules, as shown in table 3 . Moreover, for the latter both $D_{x}^{R}$ and $D_{y}^{R}$ decrease at a similar rate, and diffusion about the $y$ axis is still the predominant mechanism at the lowest analyzed temperature. Then, the decreasing rate of rotational diffusion strongly depends on the specific HB state.

\subsection{Rotational relaxation}

Theoretical approaches on rotational relaxation lead to analytical relations for the mutual dependence between relaxation time of the angular momentum and that of reorientation [15]. We have tested our results against predictions of the J-diffusion, M-diffusion and Langevin models. According to the J-diffusion approach [16], rotational dynamics is modelled as a series of collisions. Between them, molecules rotate with angular velocities according to a Boltzmann distribution. The angular momentum is randomized after each collision, both in direction and magnitude. Within the M-diffusion 
model, only the direction of the angular momentum is considered to be randomized after each collision [16]. In the Langevin model, the angular moment is assumed to follow a Langevin equation [17]. For the latter, no analytical solution exists in three dimensions, but a numerical curve for a spherical molecule can be derived [28].

The adimensional parameters $\tau_{2}^{*}=\tau_{2}\left(k_{B} T / I\right)^{1 / 2}$ and $\tau_{J}^{*}=\tau_{J}\left(k_{B} T / I\right)^{1 / 2}$ are plotted in figure 6 . For methanol, the reorientation time $\tau_{l}$ has been obtained by fitting a stretched exponential function to $C_{2}(t)$ (eq. (1)), $\tau_{J}$ has been evaluated following eq.(6) and $I$ has been approximated by the average value of the diagonal terms of the inertial tensor. Predictions corresponding to the J- and M-diffusion models for spherical molecules, as well as the numerical curve corresponding to the Langevin approach are also shown. Results obtained for a methanol-like system where HB cannot be established are also displayed in figure 6 . In the modelling of such system, hydrogen sites of the methanol model have been suppressed and, as a result, molecules have a linear geometry [9]. It is apparent that both the J-diffusion and the Langevin model reproduce reasonably well the behaviour of the latter system even at temperatures within the supercooled state. Similar degree of accord was achieved for other non-associated solvents as neopentane [29] and carbon tetrachloride [15], also shown in the figure.

It is apparent in figure 6 that none of the models predicts the results obtained for methanol at the whole range of analyzed temperatures. Results obtained for the non-associated methanol system suggest that non-sphericity of methanol molecules is not able to explain discrepancies with theoretical models, and that the existence of a HB network between molecules is probably the main reason for them. Results published elsewhere for water have also been displayed in figure $6[30,31]$. Similar trends between both solvents can be detected, in spite of the remarkable differences between their HB networks. For both water and methanol, model $\mathrm{M}$ is the best approximation at room temperature, but discrepancies with predictions of the model increase upon cooling.

\section{Concluding remarks}

Anisotropic rotational dynamics has been studied for methanol at several temperatures. It has been found that out-of-plane rotational dynamics takes place at shorter frequencies than in-plane rotations. Upon cooling, the main peaks for rotational spectra are shifted towards slightly larger frequencies. 
Moreover, shoulders observed at room temperature develop into secondary peaks, specially for in-plane rotation.

It has been found that rotational dynamics depends on the specific number of hydrogen bonds that a molecule establishes. Indeed, as it increases, the main peaks on the spectra shift towards larger frequencies, which is consistent with an increasingly constrained dynamics. Accordingly, diagonal terms of the rotational diffusion tensor are smaller for subsets of molecules that establish larger number of hydrogen bonds. All rotational diffusion coefficients decrease upon cooling, but the microscopic mechanisms for such a decrease are not the same at all temperatures. That is, whereas out-of-plane rotation of $\mathrm{CH}_{3}$ sites is the predominant mechanism for diffusional rotation at room temperature, in-plane rotation becomes most relevant at very low temperatures. But this is not true for molecules that establish one hydrogen bond. Then, the decreasing rate of rotational diffusion strongly depends on the specific hydrogen bonded state.

Rotational relaxation has also been investigated. The Langevin model predicts reasonably well the behaviour of the non-HB molecules, even in the supercooled state. For the overall system, with a majority of 2-HB molecules, theoretical models that take into account the anisotropy of dynamics are still lacking. Similar trends between methanol and water have been obtained, in spite of remarkable differences between their specific hydrogen bonded networks.

\section{Acknowledgments}

We thank Rossend Rey for interesting suggestions and for providing data for figure 6. Finantial support of MINECO (Projects FIS2015-66879-C2-1-P and PGC2018-099277-B-C21) is acknowledged. 


\section{References}

[1] P.Sindzingre and M.L.Klein, J. Chem. Phys. 92 (1992) 4681.

[2] R. Palomar and G. Sesé, J. Phys. Chem. B 109 (2005) 499.

[3] K. Yoshida, T. yamaguchi, T. Yokoo and S. Itoh, J. Mol. Liq. 222 (2016) 395.

[4] M. Matsumoto and K. E. Gubbins, J. Chem. Phys. 93 (1990) 1981.

[5] R. Palomar and G. Sesé, J. Chem. Phys. 133 (2010) 044501.

[6] E. Guàrdia, G. Sesé, and J.A. Padró, J. Mol. Liq. 62 (1994) 1.

[7] J. Martí, E. Guàrdia and J.A. Padró, J. Mol. Liq. 64 (1995) 1.

[8] A. Carrington and A.D. McLachlan. Introduction to magnetic ressonance (Chapman and Hall, London 1967).

[9] G. Sesé, J. Ortiz de Urbina and R. Palomar, J. Chem. Phys. 137 (2012) 114502 .

[10] J. Ortiz de Urbina and G. Sesé, Phys. Rev. E 94 (2016) 012605.

[11] A. Y. Zasetsky, A. S. Lileev and A. K. Lyashchenko, Mol. Phys. 108 (2010) 649.

[12] D. Laage and J. T. Hynes, Science 311 (2006) 832.

[13] D. Laage and J. T. Hynes, J. Phys. Chem. B 112 (2008) 14230.

[14] A.A. Vartia, K.R. Mitchell-Koch, G. Stirnemann and W.H. Thompson, The Journal of Physical Chemistry B 115 (2011) 12173.

[15] R. Rey, J. Chem. Phys. 129 (2008) 224509.

[16] R. E. D. McClung, J. Chem. Phys. 51 (1969) 3842.

[17] M. Fixman and K. Rider, J. Chem. Phys. 51 (1969) 2425.

[18] J. P. Ryckaert, G. Cicotti and H. J. C. Berendsen, J. Comput. Phys. 23 (1977) 327. 
[19] W. L. Jorgensen, J. Phys. Chem. 90 (1986) 1276.

[20] M.P. Allen and D.J. Tildesley. Computer Simulations of liquids (Clarendon Press, Oxford, 1987).

[21] Haughney M., Ferrario M. and McDonald I. R. J. Phys. Chem. 91 (1987) 4934.

[22] González M. A., Enciso E., Bermejo F. J. and Bée M., Phys. Rev. B 61 (2000) 6654.

[23] L. M. Svishchev and P. G. Kusalik, J. Chem. Phys. 98 (1994) 728.

[24] D. Rozmanov and P. G. Kusalik, J. Chem. Phys. 136 (2012) 044507.

[25] M. G. Mazza, N. Giovambattista, H. E. Stanley and F. W. Starr, Phys. Rev. E 76 (2007) 031203.

[26] R. M. Lynden-Bell and I. R. Mc Donald, Mol. Phys. 43 (1981) 1429.

[27] B. Guillot, P. Marteau and J. Obriot, J. Chem. Phys. 93 (1990) 6148.

[28] G. Lévi, J.P. Marsault, F. Marsault-Hérail and R.E.D. Clung, J. Chem. Phys. 73 (1980) 2443.

[29] R. Rey, J. Phys. Chem. B 112 (2008) 344.

[30] F. Sciortino, P. Gallo, P. Tartaglia and S.-H. Chen, Phys. Rev. E 54 (1996) 6331.

[31] D. Paschek and A. Geiger, J. Phys. Chem. B 103 (1999) 4139. 


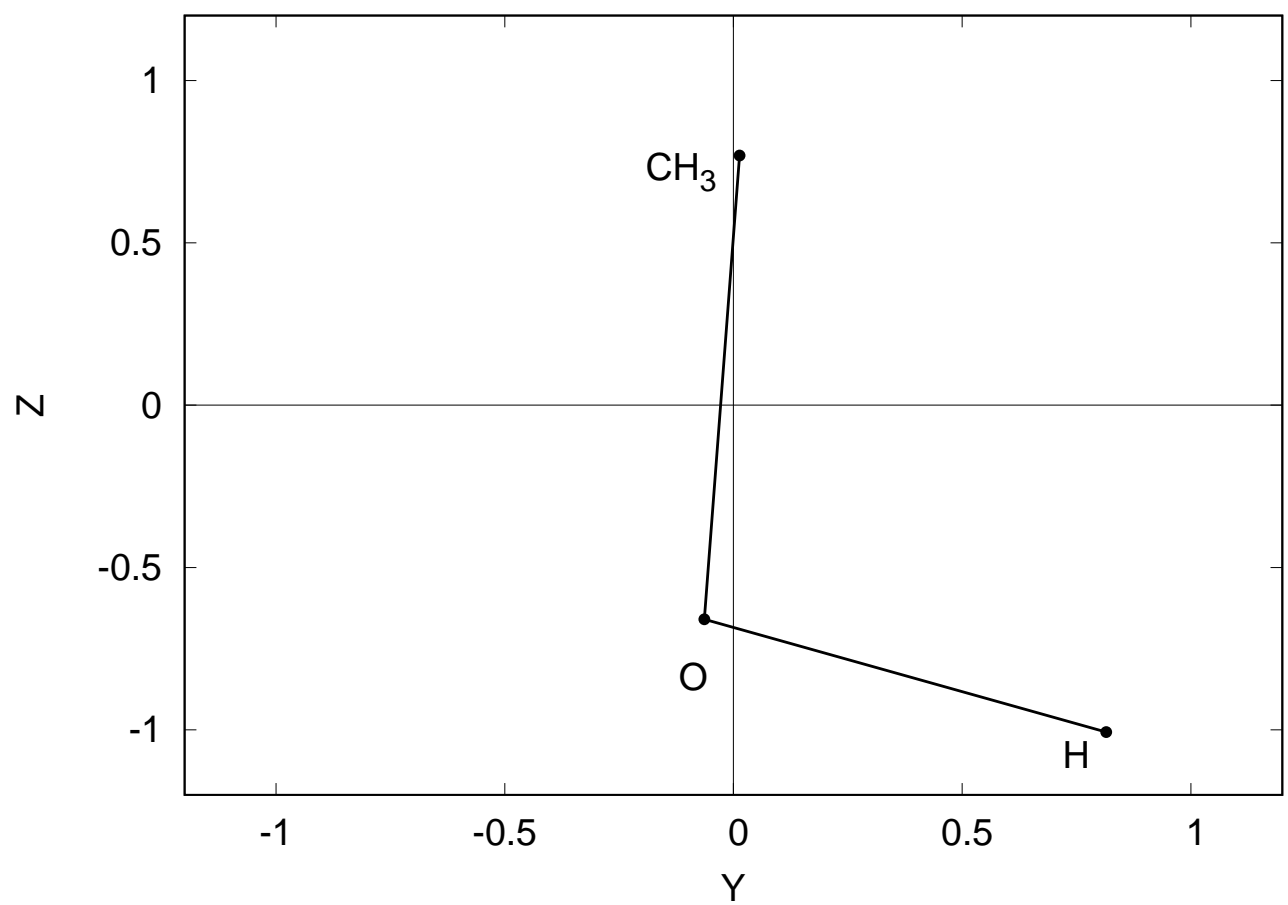

Figure 1: Scheme of the three-site methanol molecular model. Principal inertial axes are centered at the molecular center-of-mass. The molecule is located within the $y z$ plane, whereas the $x$ axis is perpendicular to the molecular plane. 


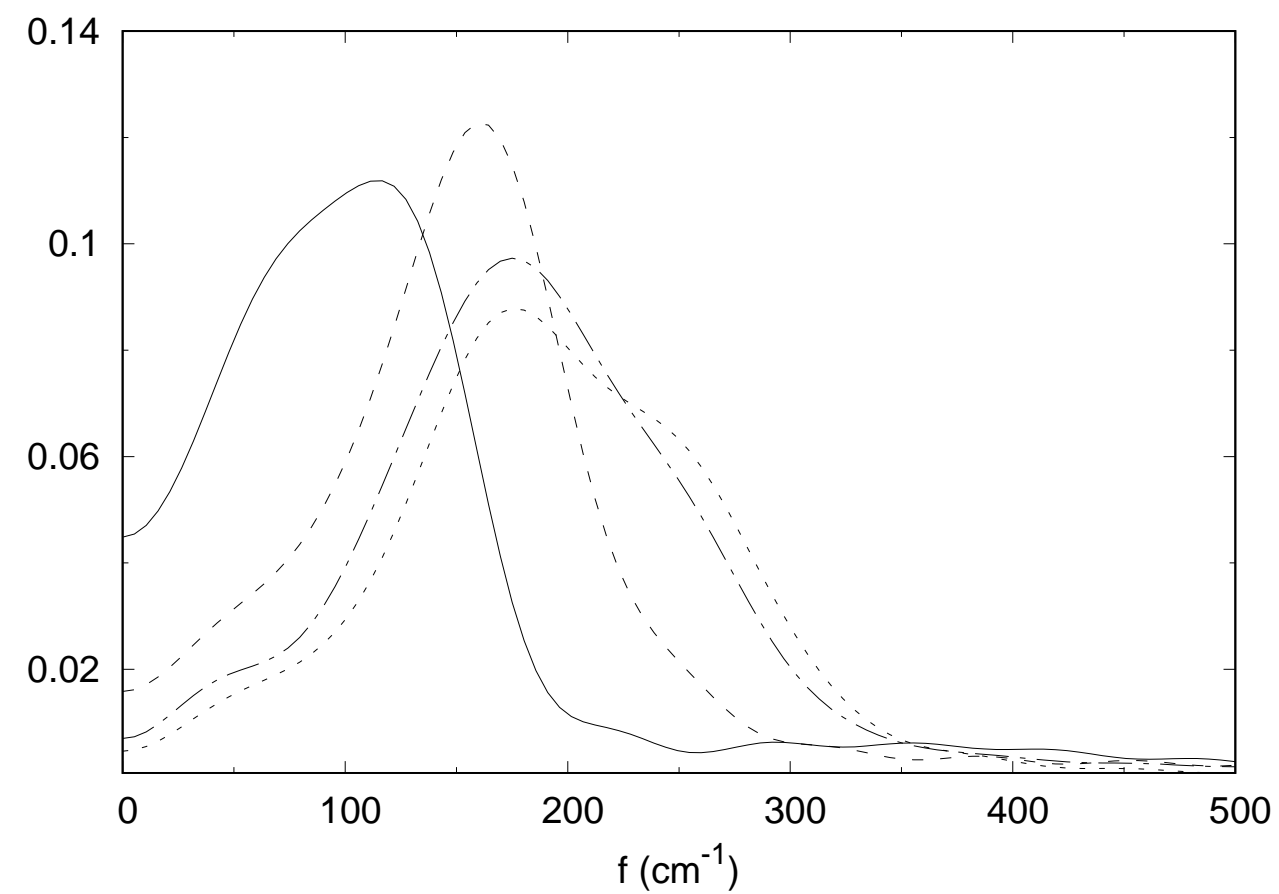

Figure 2: Spectra of $C_{\omega_{x}}(t)$ correlation functions (eq.2) at $298 \mathrm{~K}$ for molecules that establish $0 \mathrm{HB}$ (continuous line), $1 \mathrm{HB}$ (dashed line), $2 \mathrm{HB}$ (dotted-dashed line) and $3 \mathrm{HB}$ (dotted line). 


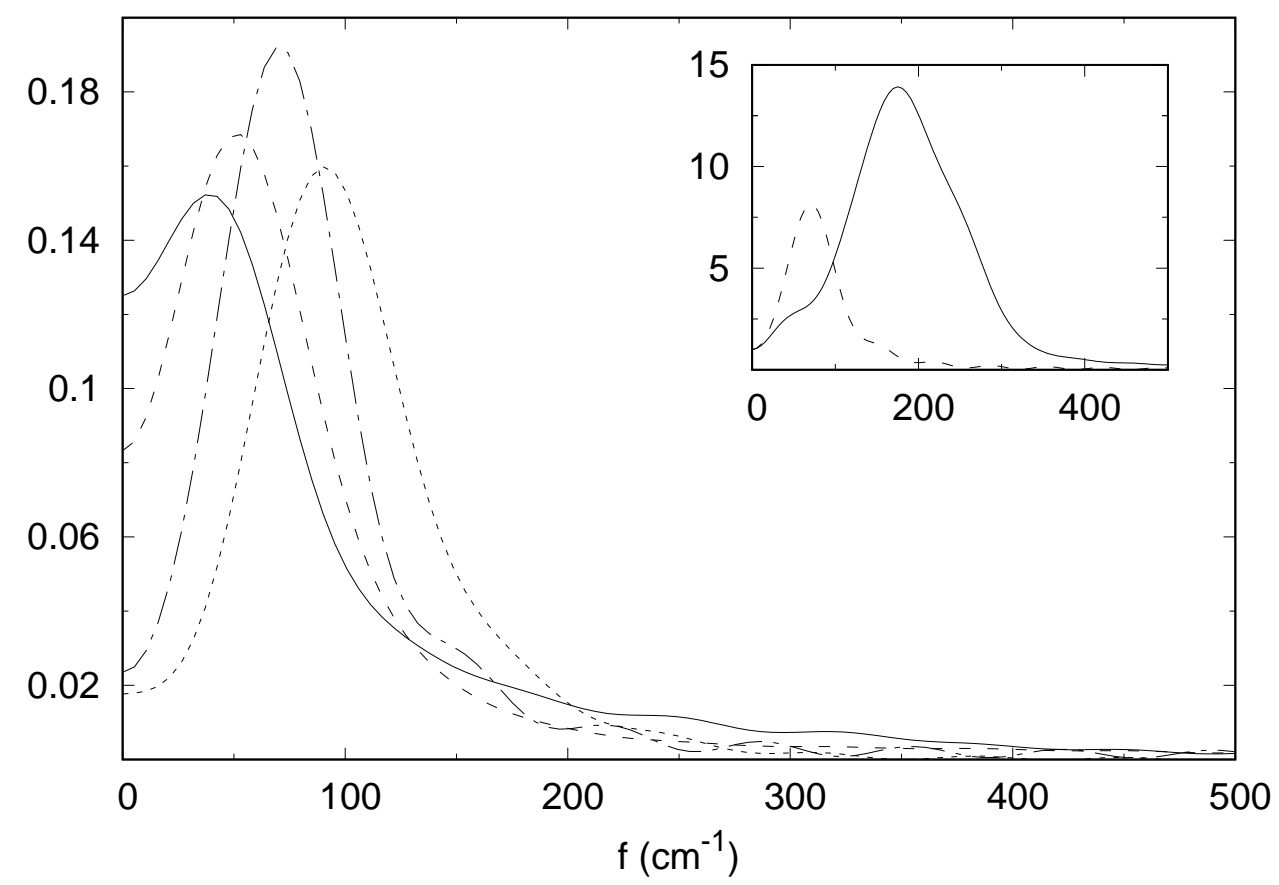

Figure 3: Spectra of $C_{\omega_{y}}(t)$ correlation functions (eq.2) at $298 \mathrm{~K}$ for molecules that establish 0 HB (continuous line), 1 HB (dashed line), 2 HB (dotted-dashed line) and $3 \mathrm{HB}$ (dotted line). Inset: $C_{\omega_{x}}(t)$ (continuous line) and $C_{\omega_{y}}(t)$ (dashed line) for 2-HB molecules. Please note that functions in the inset have been normalized so that their value at zero frequency has been set to unity. 


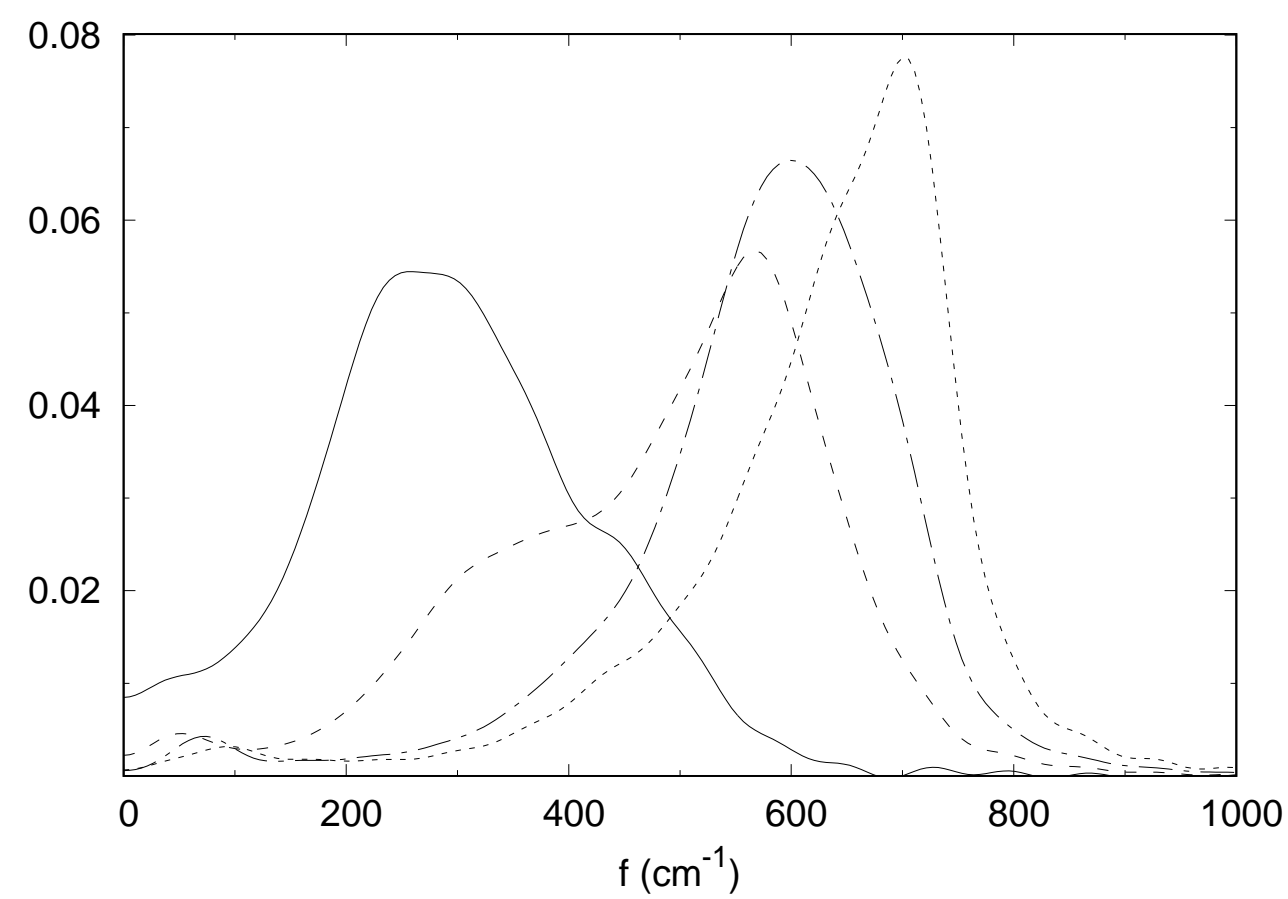

Figure 4: Spectra of $C_{\omega_{z}}(t)$ correlation functions (eq.2) at $298 \mathrm{~K}$ for molecules that establish $0 \mathrm{HB}$ (continuous line), $1 \mathrm{HB}$ (dashed line), 2 HB (dotted-dashed line) and $3 \mathrm{HB}$ (dotted line). 


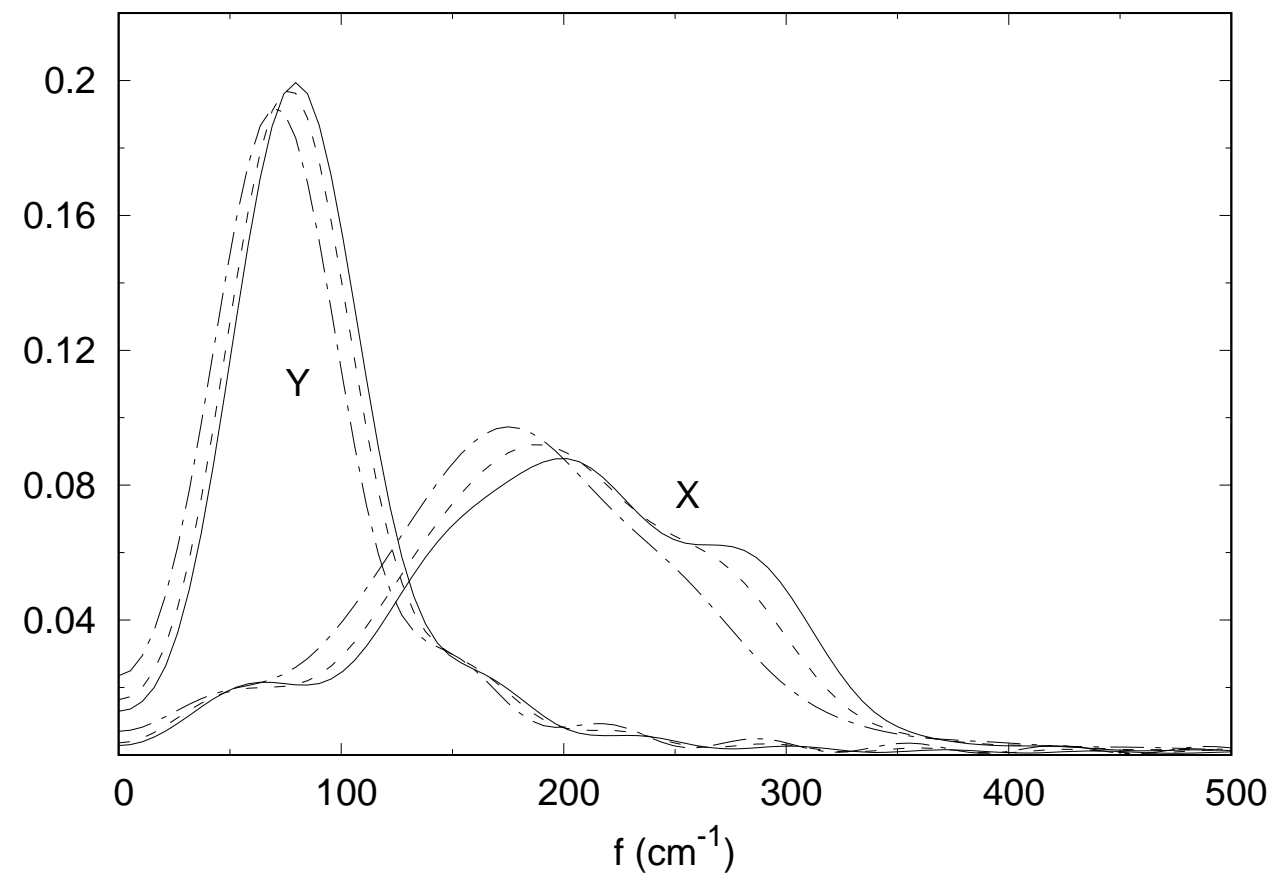

Figure 5: Spectra of $C_{\omega_{x}}(t)$ and $C_{\omega_{y}}(t)$ correlation functions (eq.2) for molecules that establish $2 \mathrm{HB}$ at $298 \mathrm{~K}$ (dotted-dashed line), $218 \mathrm{~K}$ (dashed line) and $178 \mathrm{~K}$ (continuous line). 


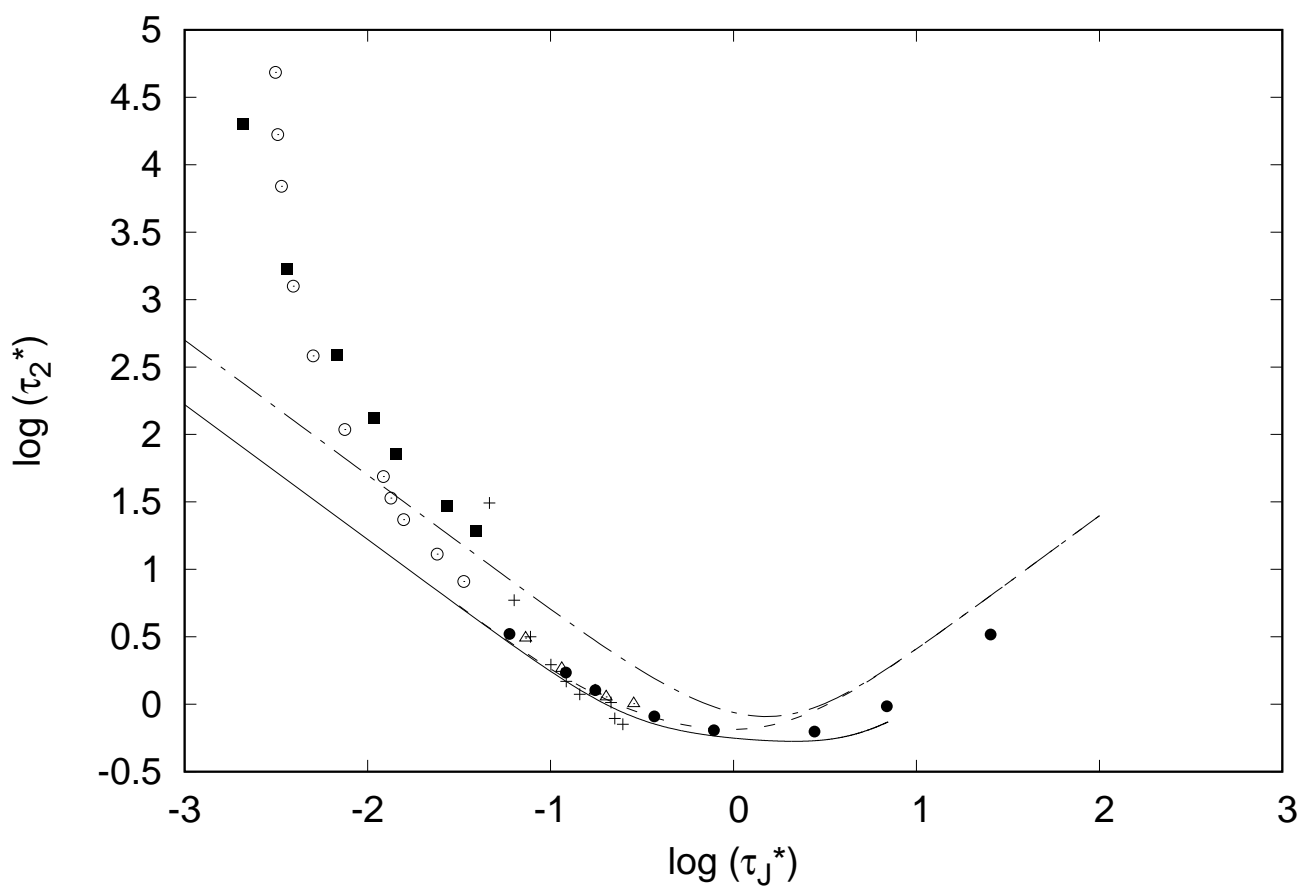

Figure 6: Reduced adimensional parameters $\tau_{2}^{*}$ vs $\tau_{J}^{*}$ for methanol (full squares), water (empty circles) (ref. [30,31]), methanol without HB (crosses) (see text for details), carbon tetracloride (filled circles) (ref. [15]) and neopentane (triangles) (ref. [29]). Also shown, theoretical curves corresponding to the Langevin (continuous line), J-diffusion (dashed line) and M-diffusion models (dashed-dotted line). 


\begin{tabular}{|c|c|c|c|c|c|c|c|}
\hline$n$ & $f_{n}$ & $D_{x}^{R}$ & $D_{y}^{R}$ & $D_{z}^{R}$ & $\tau_{x}$ & $\tau_{y}$ & $\tau_{z}$ \\
\hline 0 & $0.0126 \pm 0.0034$ & $0.322 \pm 0.009$ & $0.915 \pm 0.015$ & $1.526 \pm 0.068$ & 23. & 61. & 4.5 \\
1 & $0.1630 \pm 0.0120$ & $0.100 \pm 0.001$ & $0.562 \pm 0.006$ & $0.340 \pm 0.010$ & 7.1 & 38. & 1.0 \\
2 & $0.7560 \pm 0.0150$ & $0.045 \pm 0.001$ & $0.134 \pm 0.004$ & $0.080 \pm 0.003$ & 3.2 & 9.1 & 0.24 \\
3 & $0.0686 \pm 0.0064$ & $0.040 \pm 0.001$ & $0.098 \pm 0.003$ & $0.094 \pm 0.025$ & 2.8 & 6.7 & 0.28 \\
\hline
\end{tabular}

Table 1: Proportion of molecules sharing $n$ hydrogen bonds $\left(f_{n}\right)$, diagonal elements of their rotational tensors on the molecular reference frame (in $\mathrm{rad}^{2} / \mathrm{ps}$ ) characteristic times of angular correlation functions (in $f s$ ) at 298K. Estimated errors for the characteristic times are one unit of the last figure shown. Inertial axes of the three-site molecular model are shown in figure 1 .

\begin{tabular}{|c|c|c|c|c|}
\hline$T[K]$ & $D_{x}^{R}$ & $D_{y}^{R}$ & $D_{z}^{R}$ & $D_{y}^{R} / D_{x}^{R}$ \\
\hline 298 & $0.056 \pm 0.001$ & $0.200 \pm 0.003$ & $0.130 \pm 0.002$ & 3.6 \\
268 & $0.036 \pm 0.001$ & $0.120 \pm 0.002$ & $0.076 \pm 0.002$ & 3.3 \\
238 & $0.021 \pm 0.001$ & $0.062 \pm 0.001$ & $0.038 \pm 0.002$ & 3.0 \\
218 & $0.017 \pm 0.001$ & $0.039 \pm 0.002$ & $0.021 \pm 0.002$ & 2.3 \\
198 & $0.013 \pm 0.001$ & $0.024 \pm 0.002$ & $0.011 \pm 0.002$ & 1.8 \\
178 & $0.008 \pm 0.001$ & $0.009 \pm 0.003$ & $0.007 \pm 0.002$ & 1.1 \\
158 & $0.006 \pm 0.001$ & $0.004 \pm 0.002$ & $0.004 \pm 0.002$ & 0.7 \\
\hline
\end{tabular}

Table 2: Diagonal elements of the rotational tensor on the molecular reference frame of methanol $\left(D_{i}^{R}\right)$.

\begin{tabular}{|c|c|c|}
\hline$T[K]$ & $D_{x}^{R}$ & $D_{y}^{R}$ \\
\hline 298 & $0.100 \pm 0.001$ & $0.562 \pm 0.006$ \\
268 & $0.072 \pm 0.003$ & $0.433 \pm 0.005$ \\
238 & $0.054 \pm 0.002$ & $0.258 \pm 0.006$ \\
218 & $0.036 \pm 0.002$ & $0.203 \pm 0.011$ \\
198 & $0.027 \pm 0.002$ & $0.147 \pm 0.010$ \\
178 & $0.019 \pm 0.002$ & $0.069 \pm 0.017$ \\
158 & $0.012 \pm 0.003$ & $0.054 \pm 0.015$ \\
\hline
\end{tabular}

Table 3: Diagonal elements of the rotational tensor on the molecular reference frame of 1-HB methanol molecules. 